\title{
Genetic aspects of pain and its variability in the human population
}

\author{
Wojciech Wiesław Świtała ${ }^{1, A, D, F} \oplus$, Olga Szymańska-Adamcewicz ${ }^{2, C-D \oplus}$, Szymon Jurga ${ }^{2, A, D-E \oplus}$, \\ Emilia Pilchowska-Ujma ${ }^{2, A, C-D \oplus}$, Jan Krakowiak ${ }^{3, C-D \oplus}$
}

${ }^{1}$ Department of Anaesthesiology and Intensive Care, University Hospital, Collegium Medicum, University of Zielona Góra, Poland

${ }^{2}$ Department of Neurology, University Hospital, Zielona Góra, Collegium Medicum, University of Zielona Góra, Poland

${ }^{3}$ Faculty of Social Medicine, Medical University, Łódź, Poland

A - Research concept and design, B - Collection and/or assembly of data, C - Data analysis and interpretation, $D$ - Writing the article, E-Critical revision of the article, F - Final approval of article

\begin{abstract}
Świtała WW, Szymańska-Adamcewicz O, Jurga S, Pilchowska-Ujma E, Krakowiak J. Genetic aspects of pain and its variability in the human
\end{abstract} population. Ann Agric Environ Med. 2021; 28(4): 569-574. doi: 10.26444/aaem/134151

\section{Abstract}

The sensation of pain is common to both animals and human beings. Its threshold, intensity, tolerability, and characteristics are variable and depend on ethnicity, gender, stress exposure, co-existing mental disorders, such as depression or anxiety, social and economical background, as well as on genetic factors. It is estimated that about 5 and 20 percent of population suffer from acute and chronic pain, respectively, which results in the search for medical advice in healthcare facilities, and causes great expenses in health care budgets worldwide. Research aimed at identifying the causative agents of pain syndromes include single nucleotidepolymorphism (SNP), family history studies, twin siblings' genetic diversity studies, and recently, also a genome-wide association study (GWAS). Clinical syndromes of derangement of pain sensation are generally caused by single gene mutations (e.g. erythromelalgia and paroxysmal extreme pain disorder caused by mutations of SCN9A), but can also be associated with multiple gene mutations, as happens in migraine, fibromyalgia or hereditary sensory and autonomic neuropathies. Structural changes of proteins caused by gene mutations involve various cellular element, such as ion channels, receptors, scaffolding proteins, enzymes, transporting proteins, eventually leading to numerous clinical entities in which pain or its lack remain the leading symptoms. The sensation of pain is initiated by a stimulus, which activates the free nerve endings via chemical mediators, and the mechanical stimuli is then transmitted to the brain along the neurons and spinal tracts. Synaptic neurotransmitters and cell structures take part in this process and eventually affect the intensity of pain sensation.

\section{Key words}

genetics, pain, fibromyalgia, erythromelalgia

\section{INTRODUCTION}

The current definition of pain is 'an unpleasant sensory and emotional experience associated with actual or potential tissue damage', or described in terms of such damage. Recently, the International Association for the Study of Pain (IASP) has proposed the definition:

An aversive sensory and emotional experience typically caused by, or resembling that caused by, actual or potential tissue injury. Pain sensation is a defense mechanism required for survival, it also supports the process of damaged tissue regeneration [1].

Up-to date researche indicates that pain is the most common reason for admission and consultation in health care facilities. It is estimated that about, about $5-20 \%$ of the population suffer from acute and chronic pain, respectively, which [2] causes great expenses in healthcare budgets worldwide [3]. Among the factors influencing the threshold and intensity of pain are: environmental factors, ethnicity, gender, stress exposure, its threshold, intensity, tolerability;

Address for correspondence: Wojciech Wiesław Świtała, Department of Anaesthesiology and Intensive Care, University Hospital, Collegium Medicum, University of Zielona Góra, Zyty 26, 65-001, Zielona Góra, Poland

E-mail: puls.switala@interia.pl

Received: 22.06.2020; accepted: 09.03.2021; first published: 31.03.2021 the characteristics are variable and depend on ethnicity, gender, stress exposure, coexisting mental disorders, financial status and genetics [4]. Therefore, the threshold and intensity of pain sensation is higher among Afro-Americans compared with North American Indians. The prevalence of pain is greater among women - they demonstrate increased pain sensitivity, different response to pain treatments, and greater prevalence of chronic pain conditions, such as fibromyalgia, migraine, chronic tension-type headache, or irritable bowel disease [5].

Among the genetic causes, single mutations can account for excessive pain experience, such as that which occurs in erythromelalgia or, quite the opposite, they can totally eliminate its perception, as in the case of congenital insensivity to pain [6]. The SCN9A gene codes a protein creating an alpha- subunit of a voltage-gated sodium channel $\mathrm{NaV}$ 1,7. Its missense mutations can lead to its prolonged opening, resulting in long-standing activation of neurons, or conversely, a shortened time of channel opening with an inability to stimulate the neural transmission [7]. The first case results in intensive pain, the latter - lack of pain. Fibromyalgia and hemiplegic migraine are examples of the involvement of multiple genes in the development of each disease.

The Genome-wide Association Study (GWAS) is an observational study helpful for the assessment of poly-gene 
inheritance [8]. The current aims at presenting the latest achievements of genetics in establishing the background of some clinical pain syndromes and their variability.

Hereditary, sensory, and autonomic neuropathies (HSAN) belong to genetic disorders characterized by the impairment of pain sensation, leading to secondary mutilations, deformities of the extremities, as well as autonomic disorders. HSAN I is inherited in an autosomal dominant way, the other entities have an autosomal recessive trait [9]. Currently, two family groups with HSAN VI and HSAN VII and VIII subtypes have been described with variant mutations in the SCN1 1A and PRDM12 genes contributing to the pathology of the disease [10]. The latest research identified variant mutations in FLVCR 1 gene for heme transporter in a patient with an unclassified HSAN. To-date, 11 genes connected with HSAN entities have been identified. Genetic testing plays an important part in the differential diagnosis of hereditary neuropathies due to symptoms overlapping in particular types of HSAN.

HSAN I. The most prevalent of hereditary somatosensory and autonomic neuropathies caused by SPTCL1 gene mutations on chromosome 9q22.1-22.3 - the gene for serine palmitoyltransferase subunit taking part in sphingomyelin and ceramide synthesis. Four SPTCL1 mutations responsible for neuropathies have been identified: C133W, C133Y, VIKKD, and G387A [11]. The genetic variants of SPTCL1 cause neural environment toxicity leading to cell death. The DNMT1 gene codes a DNA- methyltransferase protein, its variants impair the function of the enzyme and eventually interfere with the liveliness of neurons. HSAN 1 develops In patients between their 20s and 40s. Typical symptomatology includes slowly progressive impairment of sensation, at first concerning pain and temperature in distal parts of extremities. In some patients, neurological examination reveals mild motor and autonomic symptoms. Paroxysms of tearing, lancinating pain of the shanks and feet are quite common. The impairment of pain can result in chronic painless feet ulcerations, arthropathies, and osteomyelitis leading to amputations and bone deformities. Electroneurography is an important diagnostic tool, in which axonal damage of the sensory fibres can be found. There is no causative treatment.

HSAN II. An autosomal recessive disease. WNK1, KIF1A and RTREG1-gene variants are responsible for the deficit of sensation. WNK1 stands for a protein kinase 1 with lysine deficiency; the gene is localized on chromosome 12p13.33. The WNK1 protein is a neurotrophic factor required for the growth and survival of sensory neurons and Schwann cells [12]. KIF1A is located on chromosome 2 q373 and is a gene for protein transporting synaptic vesicles to the synaptic junction, which takes place via microtubules of neural cells. Variants in this gene cause synaptic impairment of pain transmission. RETREG1A - locus $5 \mathrm{p} .12$ - is a gene for a Cis protein of Golgi apparatus essential for the longevity of neurons localized in nociceptive and autonomic ganglia. The disease starts in early childhood with deformities of the feet and toes. All modalities of sensation are impaired in both upper and lower extremities; sometimes, the trunk and face are also involved. The autonomic symptoms include disturbance of perspiration, tonic pupils, orthostatic hypotension and gastroesophageal reflux. The disease leads to acrodystrophic lesions and painful bone fractures. There is no causative treatment.
HSAN III. Hereditary neuropathy is also known as Riley-Day syndrome or familial dysautonomia. It is caused by variants in ELP1 gene coding an elongator complex protein $1-\mathrm{a}$ component of proteins composing cellular scaffolding and B-lymphocytic light K polypeptide gene's amplifier inhibitor located on chromosome 9q31-33. This peptide belongs to 6 proteins vital for the liveliness and mobility of cytoplasm. The symptoms are present right from birth. Lack of fungiform papillae, impairment of lacrimation, perspiration, pain and temperature sensation, dysregulation of blood pressure with severe orthostatic hypotonia are typical for this entity. Sick infants suffer from emesis, difficulties in food intake, recurrent pulmonary infections. They are usually short and have spine deformities. The disease can be life-threatening. No causative treatment is available.

HSAN IV. Also known as congenital insensitivity to pain with anhydrosi, caused by mutation in NTRK1 gene located on chromosome 1q21. The gene codes a protein being a neurotrophic tyrosine kinase 1 essential for the development and survival of neural cells participating in the transmission of pain, touch and temperature. It is located on the surface of neurons and activated in connection with $N G F-B$. The interaction between receptor (TrkA) and its ligand (NGF) during the prenatal period is crucial for the development and survival of small neurons (autonomic, pain and temperature transmition). The disease begins in infancy. Typical symptomatology includes recurrent fevers, anhydrosis, pain insensitivity, tendency for self-mutilation, mental retardation. Lack of pain leads to damage of soft tissues, painless fractures, joint damage and, in consequence, significant disability. Causative treatment is unknown $[9,13]$.

HSAN V. Hereditary sensory autonomic neuropathy type $\mathrm{V}$ is a genetic disease caused by NGF mutations - a protern for a protein, a beta growth factor of the nerves, a partner of NTRK1 binding protein. The symptoms of the disease occur as early as in infancy. The babies do not feel pain which results in soft tissue injuries, including the tongue, lips and biting the gums. Painless injuries lead to fractures, bad healing of wounds, and mutilitating acropathies. There is no retardation, perspiration disorders or fevers which enables differential diagnosis with HSAN Iv [13].

HSAN VI. Hereditary sensory autonomic neuropathy type VI has been described recently. It is caused by mutation in the DST gene for a protein called dystonine - an element of neurons' cytoskeleton, present in spinal roots' neurons, skin and skeletal muscles. The mutation damages small and large neurons leading to severe ataxia, dystonia, extremities' mutilations. The disease usually leads to death in early childhood. Cases of long survival have been described in the literature with the disease occurring in the first decade of life, with progressive mutilations resulting in joint deformities and amputations of the extremities. There were deficits of pain, touch and vibration sensation. Pupil disorders and gastrointestinal and sexual dysfunction have also been found [10].

HSAN VII. Hereditary sensory autonomic neuropathy type VII is caused by mutation of SCN11A gene on chromosome 3 p22.2. The gene codes a protein constituting an alpha2 subunit of sodium channel and is found in the perispinal 
ganglia and trigeminal ganglion [14, 15]. Among the symptoms are insensitivity to pain, excessive perspiration and gastrointestinal motoric dysfunction which results in constipations and diarrhea. Excessive sweating causes pruritus. The disease appears in infancy with injuries to the soft tissues, fractures, joint dysfunctions, weakness in skeletal muscles, slowing of motor development, and frequent infections of the lower respiratory tract. The patients require thoughtful care and treatment for the injuries incurred.

HSAN VIII. Hereditary sensory autonomic neuropathy type VIII is caused by PRDM12 gene mutation on chromosome 9q34.12 coding a protein being a transcription regulator modifying the histons' proteins in terms of proper localization of DNA strands in cells' nucleus [16]. It can be found in nociceptors and the mutation results in lack of the ability to feel pain. The disease is characterized by insensitivity to pain, temperature, reduction of lacrimation and perspiration. Clinically, injuries to the hands and feet, deep, often infected wounds of the extremities, oral cavity, throat and face, are the most prevalent. Lack of distal phalanges in hands and feet can often be observed due to them being gnawed by afflicted children. The disease is accompanied by lung infections of mild course. There is no causative treatment. Patients require care, avoidance of injuries, and treatment of the injuries incurred.

Migraine. Migraine belongs to primary headaches and is defined as a frequent chronic neuro-muscular disorder characterized by paroxysms of severe headache; in some of patients, prodromal symptoms known as aura can occur. Epidemiological research indicates that in highly developed countries migraine affects as much as $15 \%$ of the general population - women three times more often than men. The disease usually starts between 20 - 30 years of age, often coexisting with other medical conditions, such as insomnia, chronic fatigue syndrome and fibromyalgia [17]. International Headaches' classification identifies six types of migraine, with the main division being beween migraine with and without aura [18].

The etiology of migraine is still not fully understood. It is a genetically conditioned disease in which there is a predisposed sensitivity to neurovascular reactions, or to cyclic changes in the central nervous system [19]. Evidence from research indicate that the genetical factor plays an important part in migraine with aura rather than in the classic migraine - especially CACNA1A variants on chromosome 19q.13 for a protein forming an alpha subunit of the calcium channel. Mutations of this gene have been found in $50 \%$ of patients with hemiplegic migraine with autosomal dominant trait. Mutations in CACNA1E on chromosome 1q31, a gene for a protein forming an alpha-subunit of calcium channel, have been found in some patients with hemiplegic migraine [20]. Multiple mutations of this gene can result in various degrees of disorders in the function of calcium channels. The ATP1A2 gene localized on chromosome 1q23.2 codes a protein forming a subunit of alpha 2-ATP-ase of natriumpotassium pump using the cellular deposits of ATP. Variants of this gene are found in patients with migraine [19].

The SCN9A gene on chromosome 2p24 is another gene variants of which are present in patients with migraine. This gene is linked with a neuronal natrium channel for spinocerebellar ataxia [17]. A relationship between ACE
I/D gene polymorphism and the occurrence of migraine has been proven. The DD and ID genotype makes the risk for migraine with aura higher, and the ID genotype also contributes to development of migraine with aura [21]. The hereditary connections of migraine can be accounted by the fact that it constitutes an element of clinical symptomatology of MELAS (mitochondrial encephalopathy with lactic acidosis and stroke-like episodes) and CADASIL (cerebral autosomal dominant arteriopathy with subcortical infarcts and leukoencephalopathy) syndrome.

Migraine is clinically characterized by recurrent, strong, pulsating headache, most often one-sided and localized in the frontal and temporal area, accompanied by autonomic symptoms such as nausea, vomiting, light and noise hypersensitivity. According to diagnostic criteria, the headache lasts at least $4-72$ hours, with pain of significant intensity [22]. The episodes of headache lasting more than 72 hours are called status migrainosus.

Complex migraine. accounts for $10-15 \%$ of migrainerelated headaches. A migraine attack usually has 5 stages prodromal, with unspecific endocrine and gastrointestinal symptoms, aura, headache, resolution and postdrome. Aura can be represented as impairment of vision (scotoma, flashes of light, visual field deficits, geometric forms, vision loss), sensation (paresthesias, tingling, speech disturbances). The presence of paresis can indicate familial or sporadic hemiplegic migraine. Aura is totally reversible with symptoms lasting from 5 - 20 minutes. The headache appears about 60 minutes after the resolution of aura [23].

Medication for migraine aims at reduction of the pain and associated symptoms, as well as prevention of headache recurrence. To settle a therapeutic regime identification of provocative agents, frequency of attacks and the gratitude of pain is required. There are two methods of treatment: rescue management, aimed at fighting the attacks, and prophylactic: help in reduction of the frequency of attacks. In terms of rescue therapy, it is essential to take the medication as soon as possible after the occurrence of pain, and in an accurate dosage.

Fibromyalgia. Fibromyalgia belongs to the group of functional somatic pain syndromes. The most common symptoms include chronic musculoskeletal pain, sleep disorders, depression, chronic fatigue, cognitive impairment and fear [24]. The diagnosis of FM is based on the 1990 American College of Rheumatology Criteria (modified in 2010/2011 and 2016). The last modification includes recommendations of serum vitamin $\mathrm{D}$ measurement, as well as a practical tool supporting the diagnosis - the Mental Status Questionnaire [25]. The disease is diagnosed in 2 $4 \%$ of the general population, affecting up to 10 times more women than men. It usually begins between the 30 s and 50 s, the risk rising with age and education [28]. The exact cause of this disease remains unknown.

Fibromyalgia comes with myofascial pain, enthesopathies, tendonitis, bursitis and tunnel syndromes - carpal tunnel syndrome. Generalized fibromyalgia is characterized by widespread chronic pain leading to permanent distress and resulting in autonomic dysfunction, depression and fear $[26,27]$.

The latest research is aimed at the identification of AT risk factors, and understanding the pathophysiological 
mechanisms contributing to the development of chronic pain. Nowaday, it is believed that these are genetic, epigenetic and autoimmune factors that play a crucial role in the pathophysiology of fibromyalgia - they lead to small fibre neuropathy and inflammation of the nervous system [29]. Recently, numerous genetic and environmental factors have been discovered that can be linked to chronic pain development. The PRKAR2B and PRKACB genes coding cAMP-dependent protein kinases play an important part in cellular function signaling localized in loci 7q22.1 and 1p31.1, respectively. The GNG5 1p22.3, GNG2 14q22.1, GNG11 7q21.3 and GNB2 7q22.1 all code the proteins transporting signals from cell membrane to the inner effector structures. The polymorphism of these genes may interfere with the proper cellular and effector signalling [29] HTT on chromosome $4 \mathrm{p} 16.3$, which is a gene for Huntington protein - a microtubule-related transporting protein. It takes part in the transport of serotonin to neural synapses localized in the brain. Mutations of this gene impair or prevent the vesicles' transport to their destination which can be the source of chronic pain. The HTR2A gene is located on chromosome 13 q14.2 and codes a serotonin receptor protein, its mutations prevent the neurotransmitter from binding with the receptor and can account for chronic pain [28]. Most of these genes take part in the biological processes and routes connected with clinical symptoms of the disease.[29]. The other possible contributing genes are ZNF77, MYT1L, NRXN3, COMT, SLC6A4, TRPU2, TRPU3.

Lately, the hypothesisthatf fibromyalgia is a result of central sensitization has been promoted - more excessive pain sensation due to spinal and cerebral amplification of neurotransmission from pain receptors - nociceptors. The role of inflammatory mediators of IL-6, IL-8 and TNF-alpha cytokines in initiation and modulation of pain intensity has been highlighted. This draws attention to the possible role of sleeping disorders in etiopathogenesis of this entity [30]. The role of long non-coding RNA strains (LncRNA) also seems to be important - they modify interactions of the genes connected with fibromyalgia [29].

The leading symptom of fibromyalgia is a chronic pain, widespread over the whole body, with duration of over 3 months. According to the American College of Rheumatology, the pain is considered generalized if it is bilateral, localized above and beneath the waistline, and in the body axis - axial skeletal pain. The second main symptom is tenderness on palpation in 11 of 18 specific tender sites (muscle attachments or their course) [26]. Other typical syndromes include chronic fatigue, sleeping disorders, memory loss and deficit of concentration, mood disorders, fears, migraine and tension headaches, gastrointestinal dysfunction, bladder hyperactivity, mucosal dryness, Reynaud syndrome, and temporomandibular joint dysfunction [31].

Rich symptomatology and common occurrence in the population presents a real challenge fort establishing the diagnosis of fibromyalgia. Generalized, chronic pain lasting for more than 3 months, with tenderness in 11 of 18 tender point sites, were made the criteria for the American College of Rheumatology (ACR) in 1990 [31]. These criteria were replaced by new ones in 2010 when a widespread pain index (WPI) and symptom severity score (SSS) were introduced. Revision of the diagnostic criteria in 2016 also introduced some major changes: fibromyalgia should be diagnosed with the scores of WPI $>7$ and SSS $>5$ or WPI $>4-6$ and SSS $>9$.
This adds the criterium of pain localized in at least 4 out of 5 regions, as well as the fibromyalgia symptoms scale (FS) or polisymyomatic distress (PSD), and rejects the exclusion of other conditions that could otherwise explain the pain. These modifications create one set of criteria [32].

The treatment of fibromyalgia requires a multidisciplinary and comprehensive approach which includes pharmacotherapy, physiotherapy, psychotherapy, and especially cognitive behavioral therapy (CBT) and various alternative methods. There is no causative treatment and pharmacotherapy comes down to symptomatic measures. Treatment with antidepressants seems to be most efficient with both - older generation I tricyclic antidepressants like amitriptyline and new ones - serotonin reuptake inhibitors (SSRI) like fluoxetine and paroxetine and serotonin norepinephrine reuptake inhibitors, e.g. duloxetine, venlafaxine and milnacipran. Antiepileptic drugs such as gabapentin and pregabalin, and dopamine agonists like pramipexole and tramadol are also useful in the treatment of fibromyalgia [35].

Monogenic clinical syndromes of variable pain sensation. Sodium channels play an important part in the process of the transmission of pain. There are many types of sodium channels, among them voltage-dependent NaV1.7, NaV1.8 and NaV1.9 play the most important role. Proteins composing these channels coded by following genes SCN9A, SCN10A and $S C N 11 A$, respectively, are localized in the neurons of ganglia of the dorsal roots, sympathetic ganglia and neural endings. $S C N 9 A$ is a gene for a protein creating and alpha subunit of NaV1.7 sodium channel. Single nucleotide polymorphism (SNP) may result in changes of the channels sensitivity in 2 variants - the nonsense type leading to premature closing of the channel and lack of propagation of depolarization along the neuron which eventually stops the sensation of pain (insensitivity to pain); the missense type enhancing the permeability of the channel results in elongation of the depolarization time and thus causes hypersensitivity to pain (neuropathic pain) [34]. Mutation of a single nucleotide in the 1150 position of allele rs 674630 on chromosome SCN9A, leads to prolonged opening time of $\mathrm{NaV} 1.7$ sodium channel and is a reason for primary erythromelalgia and paroxysmal extreme pain disorder $[6,36]$.

Primary erythromelalgia is a rare clinical syndrome characterized by the occurrence of a triad of symptoms: paroxysmal redness, warmth and acute burning pain. These symptoms occur in various parts of the body, particularly the hands and feet. No causative treatment is known, only cooling of the extremities brings relief. The etiology of erytromelalgia is not fully understood - it is a form of neuropathy leading to paroxysmal dilation of arterioles and arteriovenous anastomoses $[36,38]$. The secondary form of erythromelalgia accompanies myeloproliferative syndromes, connective tissue diseases, insulin dependent and noninsulindependent diabetes, multiple sclerosis, infectious diseases and trauma [36].

Paroxysmal pain disorder (PEPD), previously known as familial rectal pain, is a rare hereditary disease of mainly familial and occasionally sporadic occurrence. First recorded by Hayden and Crossman in 1959, it is characterized by paroxysms of severe pain in the rectal area, eye and/or 
mandible accompanied by autonomic symptoms, such as harlequin skin redness, syncope (especially in children), seizure and bradycardia [39]. The attacks of pain usually last from several to dozens of seconds and are provoked by defecation, intestine peristaltic, yawning, eating, cold wind, minor injuries of the buttocks, rubbing the eyes and the mandible area [40]. The disease is an effect of missense mutation in SCN9A gene on chromosome $2 \mathrm{q}$ in variant lowering the depolarization threshold and elongating its duration. It has an autosomal dominant trait [30, 40]. Examination of the genome of patients suffering from PEPD showed at least 8 mutations in SCN9A ne, having a correlation with the frequency and intensity of the pain, as well as susceptibility to treatment with carbamazepine or lack of it. In most patients, CBZ has proved to be effective; otherwise, oxcarbazepine can be prescribed [40].

Small fibre neuropathy. A disease of the peripheral nerves concerning mainly or exclusively the small somatic fibres, autonomic fibres, or both kinds, leading to deficit of sensation and/or autonomic dysfunction [40]. It is caused by the malfunction of the small, myelinated A-delta fibres and non-myelinated $\mathrm{C}$ fibres. The most prevalent cause of small fibre neuropathy is diabetes. Other etiological factors include connective tissue diseases, amyloidosis, infectious diseases (HIV, HCV), celiakia, toxins, hereditary sensory and autonomic neuropathies. Quite often the causative agent is difficult to establish - the autoimmune process being the most probable mechanism.

It has been proved that SCN9A, SCN10A and SCN11A genes could be responsible for the occurrence of small fibre neuropathy by supporting fenotype hyperactivity of neurons in the dorsal root ganglia, and modification of transmission in delta A and C fibres [7, 40]. Disorders of sodium channel permeability of the nonsense type result in the deficit in pain sensation, or even lack of it. Premature closing othe sodium channel dependent on the SCN9A gene variant, and delay in $S C N 11 A$, blocks the pain transmission by inhibition of

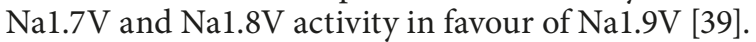

The clinical picture of small fibre neuropathy includes pain, burning and tingling sensation, and paresthesias of the distal parts of the extremities. The patients complain of discomfort in the feet. The most worrisome and typical symptom is a burning pain in the feet, reminiscent of electric shock therapy, which intensifies during the night and disturbs sleep. The symptoms gradually spread proximally towards the knees, and then involves the upper extremities distally. The involvement of autonomic fibres means the patients could feel dryness in eyes and mouth, dizzy, constipated, urine incontinence, sexual dysfunction, and abnormal sweating [38]. Small fibre neuropathy could be either disseminated or multifocal (Sjogren disease, celiakia, paraneoplastic syndromes), depending on the clinical entity [40].

The therapy for small fibre neuropathy should focus on the etiology and treatment of neuropathic pain. The majority of patients experience a slow, progressive course of the disease, with the symptoms spreading over time. They require the chronic use of painkillers. Treatment of the initial disease is important and could influence the prognosis [38, 40].

\section{CONCLUSIONS}

Clinical syndromes of derangement of pain sensation tend to occur in the early stages of life, and, in some cases, diminishing its quality and duration, for example, in hereditary insensitivity to pain. To the best of the authors' knowledge, at the moment there is no causative treatment for these syndromes, and the symptomatic treatment only improves the patients' quality of life to a minor degree. The diseases mentioned in the text constitute a significant burden for the economy of countries worldwide, as well as their healthcare budgets, because of the high expenses incurred for treatment, hospitalization, and sick leave (migraine, fibromyalgia). It is hopeed that further genetical investigation and new technologies enabling the ingerence into the human genome could contribute to the causative treatment of numerous diseases and improvement in the quality of life of patients suffering from chronic pain.

\section{REFERENCES}

1. Beckman L. The neural basis of behavior. Springer; 2012. p. 311-334.

2. Breivik H, Collett B, Ventafridda V, Cohen R, Gallacher D. Survey of chronic pain in Europe: Prevalence, impact on daily life, and treatment. Eur J Pain. 2006; 10(4): 287-287. Available from: http://doi.wiley. com/10.1016/j.ejpain.2005.06.009 [cited 2020 Oct 30].

3. Pitcher M, Von Korff M, Bushnell MC, Porter L. Prevalence and Profile of High Impact Chronic Pain in the United States. Pain. 2019; 20(2): 146-160.

4. Śliwiński Z, Starczyńska M, Śliwa M, Kiebzak W. Quality of life in patients with lumbar spinal pain. Fizjoterapia Pol. 2014; 14(2): 26-39.

5. Wiesenfeld-Hallin Z. Sex differences in pain perception. Gend Med. 2005; 2: 137-45. Available from: https://pubmed.ncbi.nlm.nih. gov/16290886/ [cited 2020 Oct 30].

6. Sharma M, Kantorovich S, Lee C, Anand N, Blanchard J, Fung ET, Meshkin B, Brenton A, Richeimer S. An observational study of the impact of genetic testing for pain perception in the clinical management of chronic non-cancer pain. J Psychiatr Res. 2017; 89: 65-72.

7. Webster LR, Belfer I. Pharmacogenetics and Personalized Medicine in Pain Management. Clin Lab Med. 2016 Sep; 36(3): 493-506.

8. Capsoni S. From genes to pain: nerve growth factor and hereditary sensory and autonomic neuropathy type V. Eur J Neurosci. 2014; 39(3): 392-400.

9. Manganelli F, Parisi S, Nolano M, et al. Novel mutations in dystonin provide clues to the pathomechanisms of HSAN-VI. Neurology 2017; 88(22): 2132-2140.

10. Wilson ER, Kugathasan U, Abramov AY, et al. Hereditary sensory neuropathy type 1-associated deoxysphingolipids cause neurotoxicity, acute calcium handling abnormalities and mitochondrial dysfunction in vitro. Neurobiol Dis. 2018: 1-14.

11. Lötsch J, Doehring A, Mogil JS, Arndt T, Geisslinger G, Ultsch A. Functional genomics of pain in analgesic drug development and therapy. Pharmacol Ther. 2013; 139(1): 60-70.

12. Axelrod FB, Kaufmann H. Hereditary Sensory and Autonomic Neuropathies. Neuromuscular Disorders of Infancy, Childhood, and Adolescence. 2nd ed. A Clinician's Approach; 2015. p. 340-352.

13. Woods CG, Babiker MOE, Horrocks I, Tolmie J, Kurth I. The phenotype of congenital insensitivity to pain due to the NaV1. 9 variant p. L811P. Eur J Hum Genet. 2015; 23: 561.

14. Phatarakijnirund V, Mumm S, McAlister WH, Novack DV, Wenkert D, Clements KL, Whyte MP. Congenital insensitivity to pain: Fracturing without apparent skeletal pathobiology caused by an autosomal dominant, second mutation in SCN11A encoding voltage-gated sodium channel 1.9. Bone 2016; 84: 289-98.

15. Chen YC, Auer-Grumbach M, et al. Transcriptional regulator PRDM12 is essential for human pain perception. Nat Genet. 2015; 47: 803-8.

16. Schmidt-Wilcke T, Diers M. New Insights into the Pathophysiology and Treatment of Fibromyalgia. Biomedicines 2017; 5(2): 22.

17. The International Classification of Headache Disorders. 3rd ed. Cephalalgia. 2013; 33: 629-808. 
18. Charles A. The pathophysiology of migraine: implications for clinical management. The Lancet Neurol. 2018; 17(2): 174-182.

19. Tabak J, Kopański Z, Kulesza-Mrowiecka M, et al. Headaches-selected epidemiological aspects and etiopatogenesis. J Clin Healthcare. 2018; 2: 23-27.

20. Sipahi T, Guldiken B, Kabayel L, Palabiyik O, Ozkan H, Kilic TO, Sut N, Turgut N. Endothelial Nitric Oxide Synthase and Angiotensin Converting Enzyme Gene Polymorphisms in Migraine Patients. Noro Psikiyatr Ars. 2013; 50(3): 274-278.

21. Fan Ch, Wolking S, et al. Early-onset familial hemiplegic migraine due to a novel SCN1A mutation. Cephalalgia. 2016; 36(13): 1238-1247.

22. Lipton RB, Silberstein SD. Episodic and chronic migraine headache: breaking down barriers to optimal treatment and prevention. Headache: J Head Face Pain. 2015; (2).

23. Akopian AN. Approaches to Cloning of Pain-Related Ion Channel Genes. Methods Mol Biol. 2013; 998: 3-19.

24. Wolfe F, Clauw DJ, Fitzcharles MA, et al. The American College of Rheumatology preliminary diagnostics criteria for fibromyalgia and measurement of symptom severity. Arthritis Care Res (Hoboken) 2010; 62(5): 600-610.

25. Eich W, Bar KJ, et al. Definition, Klassifikation klinische Diagnose und Prognose des Fibromyalgiesyndroms: Aktualisierte Leitlinie 2017 und Übersicht von systematischen Übersichtsarbeiten; Schmerz. 2017; 31(3): 231-238.

26. Fillingim RB, Smith SB, Maixner W. The phenotypic and genetic signatures of common musculoskeletal pain conditions. Nat Rev Rheumatol. 2013; 9(6): 340-50. doi: 10.1038/nrrheum.2013.43. Epub 2013 Apr 2.

27. Martí TM, Llordés ML, Jordà MC, et al. Profile of patients with fibromyalgia being treated in primary care centers in Terrassa, a city in northeastern Spain. Reumatol Clin. 2017; 13(5): 252-257.

28. Anderson RJ, McCrae CS, Staud R, et al. Predictors of clinical pain in fibromyalgia: examining the role of sleep. J Pain 2012; 13(4): 350-358.

29. Dolcino M, Tinazzi E, Puccetti A, Lunardi C. Gene Expression Profiling in Fibromyalgia Indicates an Autoimmune Origin of the Disease and Opens New Avenues for Targeted Therapy. J Clin Med. 2020; 9(6): 1814 doi: 10.3390/jcm9061814. PMID: 32532082; PMCID: PMC7356177.
30. Rusu AC, Scholich S, Meloto CB, Diatchenko L. Subgrouping of Low Back Pain Patients for Targeting Treatments Evidence from Genetic, Psychological, and Activity-related Behavioral Approaches. Clin J Pain. 2015; 31(2): 123-32.

31. Sumpton JE, Moulin DE. Fibromyalgia, neurotransmitter, pathophysiology. Handb Clin Neurol. 2014; 119: 513-527.

32. Wolfe F, Cloudw DI, Fitzcharles MA, Goldenberg DL, Häuser W, Katz RL, Mease PJ, Russell AS, Russell IJ, Walitt B. Revisions to the 2010/2011 fibromyalgia diagnostic criteria. Semin Arthritis Rheum. 2016 Dec; 46(3).

33. Kleggetveit IP, Schmidt R, Namer B, Salter H, Helås T, Schmelz M, Jørum EPathological nociceptors in two patients with erythromelalgialike symptoms and rare genetic Nav 1.9 variants. Brain Behav. 2016; 6(10).

34. Emery EC, Habib AM, Cox JJ, et al. Novel SCN9A mutations underlying extreme pain phenotypes: unexpected electrophysiological and clinical phenotype correlations. J Neurosci. 2015; 35: 7674-81.

35. Geha P, Yang Y, Estacion M, Schulman BR, Tokuno H, Apkarian VA, Dib-Haji SD, Waxman SG. Pharmacotherapy for Pain in a Family With Inherited Erythromelalgia Guided by Genomic Analysis and Functional Profiling. JAMA Neurol. 2016; 73(6): 659-667.

36. Sablonniere B, Huin V, Cuvellier J, Genet A, Dhaenens C, Vallee L. A Novel SCN9A gene Mutation in a Patient with CarbamazepineResistant Paroxysmal Extreme Pain Disorder. J Pediatric Neurol Dis. 2015; 1: 104. doi: 10.4172/2572-5203.1000104

37. Todorovic M, Haroutounian S, Dworkin R, Freeman R. Diagnostic Criteria for Idiopathic Small Fiber Neuropathy - A Systematic Literature Review. J Pain. 2019; 20(4): S118.

38. Han C, Yang Y, de Greef BT, Hoeijmakers JG, Gerrits MM, Verhamme C, Qu J, Lauria G, Merkies IS, Faber CG, Dib-Hajj SD, Waxman SG. The domain II S4-S5 linker in Nav1.9: a missense mutation enhances activation, impairs fast inactivation, and produces human painful neuropathy. Neuromolecular Med. 2015; 17: 158-69.

39. Leng XR, Qi XH, Zhou YT, Wang YP. Gain-of-function mutation p. Arg225Cys in SCN11A causes familial episodic pain and contributes to essential tremor. J Hum Genet. 2017; 62: 641-6.

40. Bennett DL, Woods CG. Painful and painless channelopathies. Lancet Neurol. 2014; 13(6): 587-99. 